\title{
On the Typical Structure of Graphs in a Monotone Property
}

\author{
Svante Janson* Andrew J. Uzzell ${ }^{\dagger}$ \\ Department of Mathematics \\ Uppsala University \\ P.O. Box 480 \\ SE-751 06 Uppsala, Sweden \\ \{svante.janson, andrew. uzzell\}@math.uu.se
}

Submitted: Apr 9, 2014; Accepted: Aug 20, 2014; Published: Aug 28, 2014

Mathematics Subject Classifications: 05C75, 05C80, 05C30

\begin{abstract}
Given a graph property $\mathcal{P}$, it is interesting to determine the typical structure of graphs that satisfy $\mathcal{P}$. In this paper, we consider monotone properties, that is, properties that are closed under taking subgraphs. Using results from the theory of graph limits, we show that if $\mathcal{P}$ is a monotone property and $r$ is the largest integer for which every $r$-colorable graph satisfies $\mathcal{P}$, then almost every graph with $\mathcal{P}$ is close to being a balanced $r$-partite graph.
\end{abstract}

Keywords: Graph limits; Monotone properties; Structure of graphs

\section{Introduction and main results}

Given a graph property $\mathcal{P}$, it is natural to study the structure of a typical graph that satisfies $\mathcal{P}$. A graph property is monotone if it is closed under taking subgraphs and hereditary if it is closed under taking induced subgraphs. Thus, every monotone property is also hereditary. Many authors have studied the structure of typical graphs in various hereditary properties - see, e.g., [1, 3, 7, 8, 9, 11], as well as the survey [4]. In this note, we use results from graph limit theory to study the structure of a typical graph in a general monotone property.

Before stating our main result, let us recall certain basic notions of graph limit theory. For more details, see, e.g., [5, 6, 13], as well as the monograph [12]. Here, we simply recall that certain sequences of graphs are defined to be convergent. A convergent sequence has a limit, called a graph limit, which is unique if it exists.

*Partly supported by the Knut and Alice Wallenberg Foundation.

${ }^{\dagger}$ Supported by the Knut and Alice Wallenberg Foundation. 
Lovász and Szegedy [13] showed that a graph limit $\Gamma$ may be represented by a graphon, a symmetric, measurable function $W:[0,1]^{2} \rightarrow[0,1]$. (So, abusing notation slightly, we will sometimes write $G_{n} \rightarrow W$ if the sequence $\left\{G_{n}\right\}_{n=1}^{\infty}$ converges to the graph limit $\Gamma$ represented by $W$.) More than one graphon may represent the same graph limit; we say that the graphons $W_{1}$ and $W_{2}$ are equivalent, and write $W_{1} \cong W_{2}$, if they represent the same graph limit.

Let $X_{1}, X_{2}, \ldots$ be i.i.d. uniformly distributed random variables in $[0,1]$. Given a graphon $W$, the $W$-random graph $G(n, W)$ is a graph with vertex set $[n]$ in which vertices $i$ and $j$ are adjacent with probability $W\left(X_{i}, X_{j}\right)$, independently of all other edges.

Let $h(x)=-x \log _{2}(x)-(1-x) \log _{2}(1-x)$ denote the binary entropy function. The entropy of a graphon $W$ is

$$
\operatorname{Ent}(W)=\int_{0}^{1} \int_{0}^{1} h(W(x, y)) \mathrm{d} \mu(x) \mathrm{d} \mu(y),
$$

where $\mu$ denotes the Lebesgue measure. As noted in [9], if $W_{1} \cong W_{2}$, then $\operatorname{Ent}\left(W_{1}\right)=$ $\operatorname{Ent}\left(W_{2}\right)$. In other words, entropy is a property of a graph limit, rather than of the graphon that represents it. Thus, we may define the entropy $\operatorname{Ent}(\Gamma)$ of a graph $\operatorname{limit} \Gamma$ to be the entropy of any graphon that represents it.

Hatami, Janson, and Szegedy [9] posed the question of which graphons may arise as limits of sequences of graphs with a given property $\mathcal{P}$. In addition to the intrinsic interest of this question, it turns out that if $\mathcal{P}$ is hereditary, then certain limits of sequences of graphs in $\mathcal{P}$ (namely, those with maximum entropy) give a great deal of information about the number and typical structure of graphs in $\mathcal{P}$. (We do not distinguish between a graph property and the class of graphs with that property.) In order to state these results, we need to introduce more notation.

Let $\mathcal{U}_{n}$ denote the set of unlabeled graphs on $n$ vertices and let $\mathcal{L}_{n}$ denote the set of labeled graphs with vertex set $[n]$. Given a graph property $\mathcal{P}$, we let $\mathcal{P}_{n}=\mathcal{P} \cap \mathcal{U}_{n}$ denote the set of unlabeled elements of $\mathcal{P}$ with $n$ vertices and let $\mathcal{P}_{n}^{L}$ denote the set of labeled elements of $\mathcal{P}$ with vertex set $[n]$. The function $n \mapsto\left|\mathcal{P}_{n}\right|$ is called the (unlabeled) speed of $\mathcal{P}$; the labeled speed is defined similarly. Observe that

$$
\left|\mathcal{P}_{n}\right| \leqslant\left|\mathcal{P}_{n}^{L}\right| \leqslant n !\left|\mathcal{P}_{n}\right|
$$

Given a graph property $\mathcal{P}$, we let $\widehat{\mathcal{P}}$ denote the set of graph limits of sequences in $\mathcal{P}$. We furthermore let $\widehat{\mathcal{P}}^{*}$ denote the set of elements of $\widehat{\mathcal{P}}$ of maximum entropy, i.e.,

$$
\widehat{\mathcal{P}}^{*}=\left\{\Gamma \in \widehat{\mathcal{P}}: \operatorname{Ent}(\Gamma)=\underset{\Gamma^{\prime} \in \widehat{\mathcal{P}}}{\max } \operatorname{Ent}\left(\Gamma^{\prime}\right)\right\}
$$

We will also use these symbols to refer to the set of graphons (respectively, the set of maximum-entropy graphons) representing limits of sequences in $\mathcal{P}$. It is shown in [9] that if $\mathcal{P}$ is hereditary (and not finite), then $\max _{\Gamma \in \widehat{\mathcal{P}}} \operatorname{Ent}(\Gamma)$ is achieved - in other words, $\widehat{\mathcal{P}}^{*}$ is nonempty. 
In [9, Theorem 1.6], Hatami, Janson, and Szegedy showed that if a hereditary property $\mathcal{P}$ has a single graph limit $\Gamma$ of maximum entropy, then a typical element of $\mathcal{P}$ is close to $\Gamma$ (in terms of the standard cut metric on the space of graph limits).

Theorem 1. Suppose that $\mathcal{P}$ is a hereditary property and that $\max _{\Gamma \in \widehat{\mathcal{P}}} \operatorname{Ent}(\Gamma)$ is attained by a unique graph limit $\Gamma_{\mathcal{P}}$. Then

(i) if $G_{n} \in \mathcal{U}_{n}$ is a uniformly random unlabeled element of $\mathcal{P}_{n}$, then $G_{n}$ converges in probability to $\Gamma_{\mathcal{P}}$ as $n \rightarrow \infty$;

(ii) if $G_{n} \in \mathcal{L}_{n}$ is a uniformly random labeled element of $\mathcal{P}_{n}^{L}$, then $G_{n}$ converges in probability to $\Gamma_{\mathcal{P}}$ as $n \rightarrow \infty$.

Now we define a special class of graphons. All of these graphons will be defined on $[0,1)^{2}$, rather than on $[0,1]^{2} ;$ it is easy to see that this change is immaterial. Given $r \in \mathbb{N}$ and $i \in[r]$, let $I_{i}=[(i-1) / r, i / r)$ and let $E_{r}=\cup_{i \neq j} I_{i} \times I_{j}$. We also let $E_{\infty}=[0,1)^{2}$. Given $r \in \mathbb{N} \cup\{\infty\}$, we let $R_{r}$ denote the set of graphons $W$ such that $W(x, y)=1 / 2$ if $(x, y) \in E_{r}$ and $W(x, y) \in\{0,1\}$ otherwise. It is easy to see that if $W \in R_{r}$, then

$$
\operatorname{Ent}(W)=\iint_{E_{r}} h(1 / 2) \mathrm{d} \mu(x) \mathrm{d} \mu(y)=\mu\left(E_{r}\right)=1-\frac{1}{r} .
$$

For $r \in \mathbb{N}$ and $0 \leqslant s \leqslant r$, we let $W_{r, s}^{*}$ denote the graphon in $R_{r}$ that equals 1 on $I_{i} \times I_{i}$ for $i \leqslant s$ and equals 0 on $I_{i} \times I_{i}$ for $s+1 \leqslant i \leqslant r$. Observe that $R_{\infty}$ consists only of the graphon that equals $1 / 2$ everywhere on $[0,1)^{2}$; for notational convenience, we denote this graphon by $W_{\infty, 0}^{*}$.

Given $r \in \mathbb{N}$ and $0 \leqslant s \leqslant r$, we let $\mathcal{C}(r, s)$ denote the class of graphs whose vertex sets can be partitioned into $s$ (possibly empty) cliques and $r-s$ (possibly empty) independent sets. In particular, $\mathcal{C}(r, 0)$ is the class of $r$-colorable graphs. Observe that for each $r$ and $s$, the class $\mathcal{C}(r, s)$ is hereditary, and that $\mathcal{C}(r, 0)$ is monotone.

It is shown in $[9$, Theorem 1.9] that if $\mathcal{P}$ is a hereditary property, then the maximum entropy of an element of $\widehat{\mathcal{P}}$ takes one of countably many values, and furthermore that this value determines the asymptotic speed of $\mathcal{P}_{n}$.

Theorem 2. If $\mathcal{P}$ is a hereditary property, then there exists $r \in \mathbb{N} \cup\{\infty\}$ such that $\max _{\Gamma \in \widehat{\mathcal{P}}} \operatorname{Ent}(\Gamma)=1-1 / r$ and such that every graph limit $\Gamma \in \widehat{\mathcal{P}}^{*}$ can be represented by a graphon $W \in R_{r}$. Moreover,

$$
\left|\mathcal{P}_{n}\right|=2^{\left(1-\frac{1}{r}+o(1)\right)\left(\begin{array}{l}
n \\
2
\end{array}\right)}
$$

Given a graph $F$, we say that a graph $G$ is $F$-free if no subgraph of $G$ is isomorphic to $F$. Given a (possibly infinite) family of graphs $\mathcal{F}$, we say that $G$ is $\mathcal{F}$-free if it is $F$-free for every $F \in \mathcal{F}$. Observe that for any family $\mathcal{F}$, the class of $\mathcal{F}$-free graphs is monotone. (Conversely, every monotone class $\mathcal{P}$ equals the class of $\mathcal{F}$-free graphs for some family $\mathcal{F}$-for example, $\mathcal{F}=\mathcal{U} \backslash \mathcal{P}$.) We write $\operatorname{Forb}(\mathcal{F})$ for the class of $\mathcal{F}$-free graphs and write $\operatorname{Forb}(F)$ when $\mathcal{F}=\{F\}$. Note in particular that $\operatorname{Forb}(\emptyset)$ equals the class of all unlabeled finite graphs, which we denote by $\mathcal{U}$. 
The coloring number of a family of graphs $\mathcal{F}$ is

$$
\operatorname{col}(\mathcal{F})=\min _{F \in \mathcal{F}} \chi(F)
$$

In particular, we define

$$
\operatorname{col}(\emptyset)=\infty
$$

Our main result says that if $\operatorname{col}(\mathcal{F})=r+1$, then a typical element of $\operatorname{Forb}(\mathcal{F})$ resembles a balanced $r$-partite graph in which cross-edges are present independently with probability $1 / 2$.

Theorem 3. Let $\mathcal{F}$ be a family of graphs and let $r=\operatorname{col}(\mathcal{F})-1$. If $\mathcal{P}=\operatorname{Forb}(\mathcal{F})$, then as $n$ tends to $\infty$, a sequence of uniformly random unlabeled (respectively, labeled) elements of $\mathcal{P}_{n}$ (respectively, elements of $\mathcal{P}_{n}^{L}$ ) converges in probability to the graph limit $\Gamma_{r}$ represented by $W_{r, 0}^{*}$.

Note that the quantity $r$ in the statement of the theorem also equals the largest integer $t$ for which every $t$-colorable graph is in $\operatorname{Forb}(\mathcal{F})$.

It follows from Theorems 2 and 3 that if $\operatorname{col}(\mathcal{F})=r+1$ then

$$
\left|\operatorname{Forb}(\mathcal{F})_{n}\right|=2^{\left(1-\frac{1}{r}+o(1)\right)\left(\begin{array}{l}
n \\
2
\end{array}\right)},
$$

which was first shown in [7]. Let us also note that Balogh, Bollobás, and Simonovits [2] obtained a fairly sharp bound on the error term in (3).

Remark 4. The proof of Theorem 3 shows that if $r=\operatorname{col}(\mathcal{F})-1$ and $\mathcal{P}=\operatorname{Forb}(\mathcal{F})$, then $W_{r, 0}^{*}$ is the unique maximum-entropy element of $\widehat{\mathcal{P}}$. For certain families $\mathcal{F}$, it is also possible to describe the set of all $\mathcal{F}$-free graph limits. For example, the set of limits of bipartite graphs is determined in [9, Example 2.1], and a very similar argument holds for $r$-partite graphs when $r \geqslant 3$. However, we know of no representation of the set of all $\mathcal{F}$-free graph limits for arbitrary $\mathcal{F}$.

Remark 5. Erdôs, Frankl, and Rödl [7] showed that if $\chi(F)=r+1$, then every $F$-free graph $G$ may be made $K_{r+1}$-free by removing $o\left(n^{2}\right)$ edges from $G$. This result is similar in spirit to Theorem 3, but we see no direct implication: if $\left\{G_{n}\right\}_{n=1}^{\infty}$ is a sequence of uniformly random $F$-free graphs and $\left\{G_{n}^{\prime}\right\}_{n=1}^{\infty}$ is the sequence of resulting $K_{r+1}$-free graphs, then the distribution of $G_{n}^{\prime}$ need not be uniform in $\operatorname{Forb}\left(K_{r+1}\right)_{n}$.

Remark 6 . Theorem 3 says that if $\operatorname{col}(\mathcal{F})=r+1$ then almost every (labeled or unlabeled) $\mathcal{F}$-free graph is close to a balanced $r$-partite graph. (Conversely, every $r$-partite graph is trivially $\mathcal{F}$-free.) In the case of labeled graphs, Prömel and Steger [14] proved a much stronger result for a specific class of monotone properties: they characterized the graphs $F$ for which almost every labeled $F$-free graph is $(\chi(F)-1)$-partite. Given a graph $F$, we say that $e \in E(F)$ is critical if $\chi(F-e)<\chi(F)$. Prömel and Steger showed that if $\chi(F)=r+1$ then

$$
\left|\operatorname{Forb}(F)_{n}^{L}\right|=(1+o(1))\left|\mathcal{C}(r, 0)_{n}^{L}\right|
$$


if and only if $F$ contains a critical edge. They also showed that if $F$ does not contain a critical edge, then there exists a constant $c_{r}>0$ not depending on $F$ such that

$$
\left|\operatorname{Forb}(F)_{n}^{L}\right| \geqslant c_{r} n\left|\mathcal{C}(r, 0)_{n}^{L}\right|
$$

for all $n$ sufficiently large. Theorem 3 shows that if $\mathcal{F}$ is any family of graphs with $\operatorname{col}(\mathcal{F})=r+1$, then $\operatorname{Forb}(\mathcal{F})^{L}$ and $\mathcal{C}(r, 0)^{L}$ have roughly the same asymptotic speed. Note that this result does not contradict (4) when $\mathcal{F}=\{F\}$ and $F$ does not contain a critical edge: if $\chi(F)=r+1$, then, in view of (1) and (3), Theorem 3 implies the weaker statement that $\left|\operatorname{Forb}(F)_{n}^{L}\right|$ and $\left|\mathcal{C}(r, 0)_{n}^{L}\right|$ differ by a factor of $2^{o\left(n^{2}\right)}$.

\section{Proof of Theorem 3}

Lemma 7. Let $\mathcal{P}$ be a monotone property and let $W \in \widehat{\mathcal{P}}$. If $W^{\prime}$ is a graphon such that $W^{\prime} \leqslant W$ pointwise, then $W^{\prime} \in \widehat{\mathcal{P}}$.

Proof. Consider the sequences of random graphs $\{G(n, W)\}_{n=1}^{\infty}$ and $\left\{G\left(n, W^{\prime}\right)\right\}_{n=1}^{\infty}$. Since $W^{\prime} \leqslant W$ pointwise, a standard argument shows that the two sequences can be coupled so that for each $n, G\left(n, W^{\prime}\right) \subseteq G(n, W)$ almost surely. It is shown in [10, Theorem 3.1] that if $W \in \widehat{\mathcal{P}}$ then, for each $n, G(n, W) \in \mathcal{P}$ almost surely. It follows that for each $n$, we almost surely have $G\left(n, W^{\prime}\right) \in \mathcal{P}$, as well. Finally, it is shown in [5, Theorem 4.5] that $G\left(n, W^{\prime}\right) \rightarrow W^{\prime}$ almost surely as $n \rightarrow \infty$, which implies that $W^{\prime} \in \widehat{\mathcal{P}}$, as claimed.

Now we prove our main result, Theorem 3.

Proof of Theorem 3. We begin by showing that, up to equivalence of graphons, $\widehat{\mathcal{P}}$ contains a unique element of maximum entropy. By Theorem 2, there exists $t \in \mathbb{N} \cup\{\infty\}$ such that $\widehat{\mathcal{P}}^{*} \subseteq R_{t}$ up to equivalence of graphons.

First, suppose that $t<\infty$. Observe that if $W \in \widehat{\mathcal{P}}^{*} \cap R_{t}$, then $W \geqslant W_{t, 0}^{*}$ pointwise, which by Lemma 7 implies that $W_{t, 0}^{*} \in \widehat{\mathcal{P}}^{*}$. We claim that, up to equivalence of graphons, $W_{t, 0}^{*}$ is in fact the only maximum-entropy element of $\widehat{\mathcal{P}}$. Indeed, let $W^{\prime} \in \widehat{\mathcal{P}}^{*} \cap R_{t}$ and suppose that $\mu\left(W^{\prime}=1\right)>0$. But then Lemma 7 implies that $\min \left\{W^{\prime}, 1 / 2\right\}$ is a graphon in $\widehat{\mathcal{P}}$ with entropy strictly larger than $1-1 / t$, which contradicts the definition of $t$.

Now we show that $t=\operatorname{col}(\mathcal{F})-1=r$. Suppose that $t<\infty$. It is observed in $[9$, Remark 1.10] that if $\mathcal{P}$ is hereditary and $0 \leqslant s \leqslant r<\infty$, then $W_{r, s}^{*} \in \widehat{\mathcal{P}}$ if and only if $\mathcal{C}(r, s) \subseteq \mathcal{P}$. By the definition of $\operatorname{col}(\mathcal{F})$, it is easy to see that if $u \leqslant r$, then $\mathcal{C}(u, 0) \subseteq \mathcal{P}$ and hence $W_{u, 0}^{*} \in \widehat{\mathcal{P}}$. On the other hand, $\mathcal{F}$ contains some element of $\mathcal{C}(r+1,0)$, which implies that $\mathcal{C}(r+1,0) \nsubseteq \mathcal{P}$. This implies that $W_{u, 0}^{*} \notin \widehat{\mathcal{P}}$ when $u \geqslant r+1$, and hence that $t=r$.

If $t=\infty$, then we claim that $\mathcal{P}=\operatorname{Forb}(\emptyset)=\mathcal{U}$; the conclusion then follows from (2). Suppose to the contrary that $\mathcal{P}$ does not contain some graph $F$. Then $\mathcal{C}(\chi(F), 0) \nsubseteq \mathcal{P}$, which implies that $W_{\chi(F), 0}^{*} \notin \widehat{\mathcal{P}}$. However, $W_{\chi(F), 0}^{*} \leqslant W_{\infty, 0}^{*}$ pointwise, so Lemma 7 implies that $W_{\infty, 0}^{*} \notin \widehat{\mathcal{P}}$, which is a contradiction. 
Finally, since $\mathcal{P}$ is a hereditary property, it follows from Theorem 1 that a uniformly random (labeled or unlabeled) element of $\mathcal{P}$ converges in probability to $\Gamma_{r}$, as claimed.

\section{References}

[1] N. Alon, J. Balogh, B. Bollobás, and R. Morris. The structure of almost all graphs in a hereditary property. J. Combin. Theory Ser. B, 101(2):85-110, 2011.

[2] J. Balogh, B. Bollobás, and M. Simonovits. The number of graphs without forbidden subgraphs. J. Combin. Theory Ser. B, 91(1):1-24, 2004.

[3] J. Balogh, B. Bollobás, and M. Simonovits. The typical structure of graphs without given excluded subgraphs. Random Structures Algorithms, 34(3):305-318, 2009.

[4] B. Bollobás. Hereditary and monotone properties of combinatorial structures. In Surveys in Combinatorics 200\%, volume 346 of London Math. Soc. Lecture Note Ser., pages 1-39. Cambridge Univ. Press, Cambridge, 2007.

[5] C. Borgs, J.T. Chayes, L. Lovász, V.T. Sós, and K. Vesztergombi. Convergent sequences of dense graphs I: Subgraph frequencies, metric properties and testing. Adv. Math., 219(6):1801-1851, 2008.

[6] P. Diaconis and S. Janson. Graph limits and exchangeable random graphs. Rend. Mat. Appl. (7), 28(1):33-61, 2008.

[7] P. Erdôs, P. Frankl, and V. Rödl. The asymptotic number of graphs not containing a fixed subgraph and a problem for hypergraphs having no exponent. Graphs Combin., 2(1):113-121, 1986.

[8] P. Erdôs, D.J. Kleitman, and B.L. Rothschild. Asymptotic enumeration of $K_{n}$-free graphs. In Colloquio Internazionale sulle Teorie Combinatorie (Rome, 1973), Tomo II, pages 19-27. Atti dei Convegni Lincei, No. 17. Accad. Naz. Lincei, Rome, 1976.

[9] H. Hatami, S. Janson, and B. Szegedy. Graph properties, graph limits, and entropy. Preprint, arXiv:1312.5626, 2013.

[10] S. Janson. Graph limits and hereditary properties. European J. Combin., to appear.

[11] S. Janson and A.J. Uzzell. On string graph limits and the structure of a typical string graph. Preprint, arXiv:1403.2911, 2014.

[12] L. Lovász. Large networks and graph limits, volume 60 of Amer. Math. Soc. Colloq. Publ. Amer. Math. Soc., Providence, RI, 2012.

[13] L. Lovász and B. Szegedy. Limits of dense graph sequences. J. Combin. Theory Ser. B, 96(6):933-957, 2006.

[14] H.J. Prömel and A. Steger. The asymptotic number of graphs not containing a fixed color-critical subgraph. Combinatorica, 12(4):463-473, 1992. 